Trinity University

Digital Commons@ Trinity

Biology Faculty Research

Biology Department

9-2011

\title{
Relationships Among Reproductive Morphology, Behavior, and Testosterone in a Natural Population of Green Anole Lizards
}

\author{
Michele A. Johnson \\ TrinityUniversity, mjohnso9@trinity.edu \\ R. E. Cohen \\ J. R. Vandecar \\ J.Wade
}

Follow this and additional works at: https://digitalcommons.trinity.edu/bio_faculty

Part of the Biology Commons

\section{Repository Citation}

Johnson, M.A., Cohen, R.E., Vandecar, J.R., \& Wade, J. (2011). Relationships among reproductive morphology, behavior, and testosterone in a natural population of green anole lizards. Physiology \& Behavior, 104(3), 437-445. doi:10.1016/

j.physbeh.2011.05.004

This Article is brought to you for free and open access by the Biology Department at Digital Commons @ Trinity. It has been accepted for inclusion in Biology Faculty Research by an authorized administrator of Digital Commons @ Trinity. For more information, please contact jcostanz@trinity.edu. 
Provided for non-commercial research and education use. Not for reproduction, distribution or commercial use.

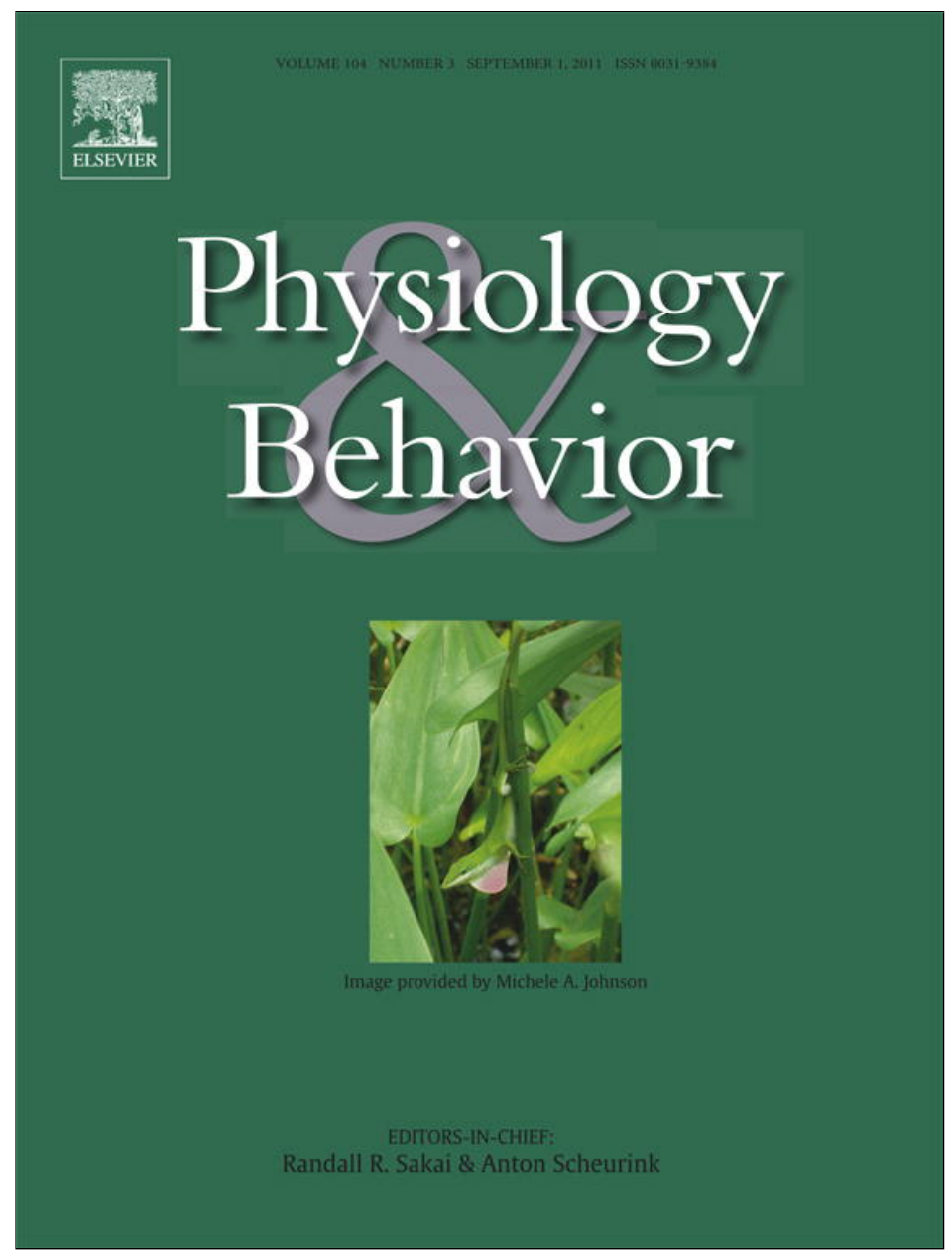

This article appeared in a journal published by Elsevier. The attached copy is furnished to the author for internal non-commercial research and education use, including for instruction at the authors institution and sharing with colleagues.

Other uses, including reproduction and distribution, or selling or licensing copies, or posting to personal, institutional or third party websites are prohibited.

In most cases authors are permitted to post their version of the article (e.g. in Word or Tex form) to their personal website or institutional repository. Authors requiring further information regarding Elsevier's archiving and manuscript policies are encouraged to visit:

http://www.elsevier.com/copyright 


\title{
Relationships among reproductive morphology, behavior, and testosterone in a natural population of green anole lizards
}

\author{
Michele A. Johnson ${ }^{\mathrm{a}, \mathrm{b}, *}$, Rachel E. Cohen ${ }^{\mathrm{b}}$, Joseph R. Vandecar ${ }^{\mathrm{c}}$, Juli Wade ${ }^{\mathrm{b}, \mathrm{c}, \mathrm{d}}$ \\ ${ }^{a}$ Trinity University, Department of Biology, One Trinity Place, San Antonio, TX 78212 USA \\ ${ }^{\mathrm{b}}$ Michigan State University, Department of Zoology, 203 Natural Science Building, East Lansing, MI 48824 USA \\ c Michigan State University, Department of Psychology, 240C Psychology Building, East Lansing, MI 48824 USA \\ ${ }^{d}$ Michigan State University, Neuroscience Program, 108 Giltner Hall, East Lansing, MI 48824 USA
}

\section{A R T I C L E I N F O}

\section{Article history:}

Received 22 February 2011

Received in revised form 11 April 2011

Accepted 3 May 2011

\section{Keywords:}

Copulation

Courtship

Dewlap

Hemipenes

Sex difference

Testosterone

\begin{abstract}
A B S T R A C T
Laboratory studies of reproductive systems have long supported the idea that neural and/or muscular structures used frequently are often enhanced in size. However, field studies integrating behavioral, morphological, and hormonal data are needed to better understand relationships in natural environments. We examined a natural population of green anole lizards (Anolis carolinensis) to determine whether variation in reproductive morphology both within and between the sexes paralleled differences in courtship and copulatory behaviors and circulating testosterone levels. Display rate in males was positively correlated with the sizes of the cartilage supporting the dewlap (a throat fan used in courtship and aggression) and renal sex segments (portions of the kidney that function similarly to the mammalian prostate), but correlated negatively with seminiferous tubule size. Plasma testosterone in males was negatively correlated with display behavior and was not correlated with any measures of morphology. Females, which display rarely, exhibited no relationships between morphology and frequency of behavior. Comparisons between the sexes show that males have consistently larger courtship and copulation morphologies than females, even when accounting for sex differences in body size. The results not only support the idea of relationships between increased function and enhanced structures, but also show the complexity of mechanistic interactions associated with reproductive behavior in wild animals.
\end{abstract}

(c) 2011 Elsevier Inc. All rights reserved.

\section{Introduction}

Successful reproduction requires a suite of specialized morphological and physiological traits, as well as the performance of stereotyped behaviors demonstrating an animal's motivation to copulate [1]. Thus, examinations of reproductive morphology and behavior offer excellent opportunities to investigate relationships between structure and function (reviewed in [2]). Studies across diverse taxa document that frequently-used reproductive structures, particularly required muscles and components of the nervous system, are often larger than those used more rarely. This relationship is clearly observed in comparisons between the sexes. For example, in species where males perform complex vocal courtship displays but females do not [e.g., zebra finches (Taeniopygia guttata), African clawed frogs (Xenopus laevis), and midshipmen fish (Poricthys

\footnotetext{
* Corresponding author at. Trinity University, Department of Biology, One Trinity Place, San Antonio, TX 78212 USA. Tel.: + 12109998918 ; fax: + 12109997229.

E-mail addresses: michele.johnson@trinity.edu (M.A. Johnson), cohenra2@msu.edu (R.E. Cohen), jrvandecar@yahoo.com (J.R. Vandecar), wadej@msu.edu (J. Wade).
}

notatus)], the neuromuscular structures supporting these behaviors are larger in males than females [3-7]. Both developmental organization and adult maintenance of these sexual dimorphisms are often facilitated by steroid hormones (reviewed in [8]).

Many studies have examined the relationships between reproductive behavior and morphology in the lab (reviewed in multiple chapters of [9]), and many others have investigated associations between sex steroid hormone levels and behavior in the lab [e.g., 10] or field [e.g., 11-13]. However, relatively little work in reproductive biology has focused on the associations among neuromuscular, endocrine, and behavioral traits within individual animals in their natural habitats.

The green anole (Anolis carolinensis) is an arboreal lizard that reproduces seasonally, from approximately April to July in the southeastern US [14-16]. Decades of field research on this species has provided extensive information regarding its behavior and reproductive ecology [e.g., 17,18]. During the breeding season, males court females and defend territories with displays that include head-bobs, push-ups, and extensions of a reddish-pink throat fan called a dewlap [19] (Fig. 1). Females have much smaller dewlaps than males, and they also perform these displays to defend territories, but at much lower rates than males [20,21]. Dewlap displays are 


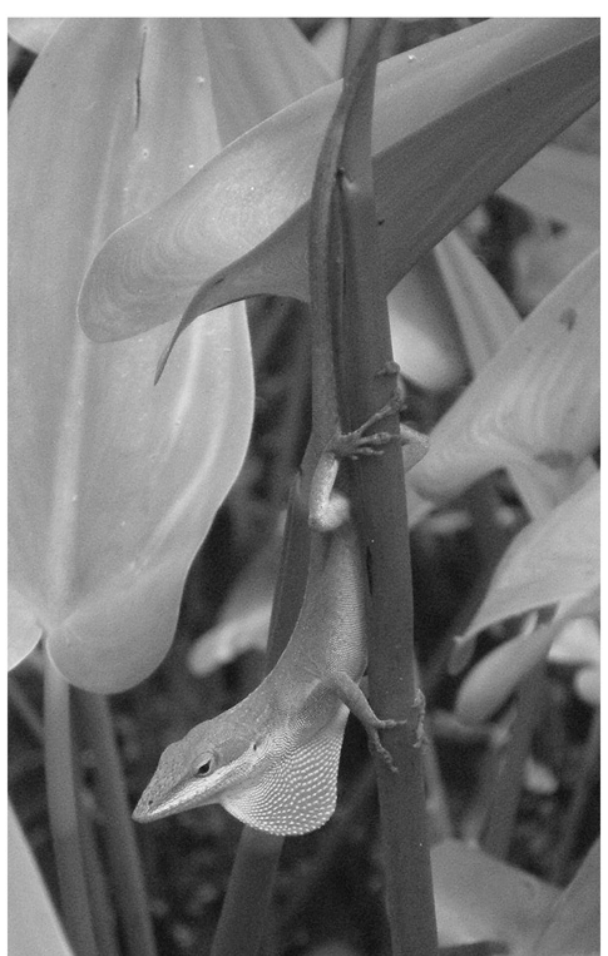

Fig. 1. Male A. carolinensis with extended dewlap (Jean Lafitte National Park, Barataria, Louisiana, USA).

important for courtship and mate selection, as well as territory defense $[22,23]$. Male courtship generally (and perhaps necessarily) precedes copulation, which occurs when a male mounts a female, maneuvers his tail under hers, everts one of two bilateral copulatory organs called hemipenes, intromits into her cloacal vent, and ejaculates [16]. These male reproductive behaviors are facilitated by increased androgen, primarily $\mathrm{T}$ (rather than its metabolites), during the breeding season [24-27]. Receptivity is activated by ovarian hormones, and while neural metabolism of $\mathrm{T}$ into estradiol may facilitate this behavior [25], specific roles of circulating $\mathrm{T}$ itself in females are not clear [28].

The neuromuscular structures supporting the movements of the dewlap and hemipenes have been well-characterized in green anoles (reviewed in [28]). The dewlap extends when the ceratohyoid $(\mathrm{CH})$ muscles on each side of the throat contract, causing the second ceratobranchial cartilage to bow out, revealing the flap of red skin of the dewlap [29,30]. Movements of the hemipenes are controlled by two muscles, the transversus penis (TPN) and the retractor penis magnus (RPM). The former wraps over the hemipenis and facilitates its eversion, and the latter is attached to the caudal end of each hemipenis as it lies in the tail and causes its retraction [31].

The goal of the present study was to build on and begin to merge existing literatures on the behavioral ecology (from the field) and relationships between behavior and morphology (from the lab) in green anoles. While field studies examine animals in their natural environments, the complexity of these environments may limit our ability to identify causal relationships among traits; yet, the simpler environments of laboratory studies may constrain our ability to extrapolate findings to the natural world. Thus, these two approaches are complementary. Although it was not the primary focus of our experiment, we evaluated circulating $\mathrm{T}$ in males to begin to assess whether relationships between this hormone and behavior, and particularly $\mathrm{T}$ and morphology, detected in the lab were observed in unmanipulated wild animals.

\section{Materials and methods}

\subsection{Field data collection}

We conducted behavioral observations on adult green anole lizards (18 males, snout-vent-length $[\mathrm{SVL}]=58-68 \mathrm{~mm}$, and 20 females, $\mathrm{SVL}=42-59 \mathrm{~mm}$ ) in Jean Lafitte National Park, Barataria Preserve in Marrero, Louisiana (N 29 47.22 , W 90 06.53) in May 2008. We located animals between $08: 30$ and 18:00 by walking through the forest until finding an apparently undisturbed lizard. During each observation (males: 64-178 min, average $131 \mathrm{~min}$; females: $22-180 \mathrm{~min}$; average $113 \mathrm{~min}$ ), we recorded all display behaviors (dewlap extensions, head-bobs, and push-ups) and determined the proportion of time the lizard displayed during the observation period. Because these displays do not obviously differ with social context (territorial or courtship displays), any dewlap extension was counted as a display bout. We also recorded all locomotor behaviors (crawls, runs, and jumps), prey captures, and copulations. As copulation was observed rarely, we were unable to include it as a variable in subsequent statistical analyses. Immediately following each observation, we captured the lizard with a noose. Because the primary focus of this study was to elucidate naturally occurring relationships between behavior and morphology, if observations of other lizards were in progress, we kept the captured lizard in an air-filled clear plastic bag until we could process it. Lizards were held for an average of 56 min (maximum of $3 \mathrm{~h}$ ).

For each lizard, the same person took the following external measurements: SVL (measured with a ruler), mass (using a Pesola spring scale), and the length of the second ceratobranchial cartilage (hereafter, 'cartilage') as seen under the skin (measured with digital calipers). Animals were then rapidly decapitated and the brain, kidneys, and gonads harvested. We also collected the portion of the throat that contains $\mathrm{CH}$ muscles and the portion of the tail with the hemipenes and RPM. All tissues were immediately frozen on dry ice until transported to Michigan State University where they were stored at $-80^{\circ} \mathrm{C}$. We collected blood from the head and trunk of males, and stored it on ice in the field. Within $8 \mathrm{~h}$, we centrifuged the samples and froze the plasma on dry ice until it was transported to Michigan State University and stored at $-80^{\circ} \mathrm{C}$.

All procedures were performed in accordance with the guidelines of the Michigan State University Institutional Animal Care and Use Committee, with permits from the National Park Service (permit \# JELA-2008-SCI-003) and the Louisiana Department of Wildlife and Fisheries (permit \# LNGP-08-059).

\subsection{Histology}

Frozen tissues were sectioned at $20 \mu \mathrm{m}$, stained with hematoxylin and eosin, and measured using Scion (NIH) Image software. For each tissue measured, we calculated an average per individual for use in statistical analyses.

In the throat, we measured cross-sectional areas of 25 arbitrarily selected fibers in the CH and GG muscles (as in $[21,32,33]$ ). The GG is a muscle involved in tongue extension, located in the throat near the $\mathrm{CH}$. Values were obtained from one section for both the left and right sides within the middle third of the rostro-caudal extent of each muscle. We also measured the cross-sectional area of the cartilage and trachea in 5 tissue sections in the middle third of the muscle [21]. Because they are in the same sections of tissue but are not involved in dewlap extension, we used the cross-sectional areas of GG fibers and the trachea as controls for general differences in body size (as in $[21,33])$.

In the tails, we measured the cross-sectional areas of 25 arbitrarily selected muscle fibers of the RPM and, as a control, the caudofemoralis $(\mathrm{CF})$, a muscle involved in leg movement that lies near the hemipenes on each side of the tail. Values for these muscles were obtained from 
one section of each side, posterior to the end of the hemipenes. Relative hemipenis size was determined by measuring the crosssectional area of the tissue on each side of the tail every $400 \mu \mathrm{m}$ from the rostral end of the hemipenes until RPM fibers were interdigitated with hemipenis tissue (indicating the caudal extent of the hemipenis).

We also measured the height of epithelial cells of renal sex segments in the kidneys (which function similarly to the mammalian prostate, and are often used as a bioassay for androgen levels [33]) and the cross-sectional areas of seminiferous tubules in the testes using 10 arbitrarily selected structures from a single section of each tissue type. We confirmed the reproductive status of all males by the presence of mature sperm in the testes and of all females by the presence of at least one yolking follicle.

\subsection{Radioimmunoassay}

T concentrations from male plasma samples were evaluated in three assays. Intra-assay coefficients of variation were $10.9 \%$ or less, and the inter-assay coefficient of variation was $12.1 \%$. Samples were incubated overnight at $4{ }^{\circ} \mathrm{C}$ with $1000 \mathrm{CPM}$ of $3 \mathrm{H}-\mathrm{T}(80.4 \mu \mathrm{Ci} / \mathrm{mL}$; PerkinElmer, Boston, MA) for recovery determination. The samples were extracted twice with diethyl ether and dried under nitrogen, then reconstituted with $500 \mu \mathrm{L}$ of $10 \%$ ethyl acetate: iso-octane and stored at $4{ }^{\circ} \mathrm{C}$ overnight. To remove dihydrotestosterone, which has limited effect on male sexual behaviors [26] (see Introduction), we ran samples through columns that contained water $(3 \mathrm{~g}$ celite: $1 \mathrm{~mL}$ water) and glycol phases ( $2 \mathrm{~g}$ celite: $1 \mathrm{~mL}$ of 1:1 propylene glycol: ethylene glycol). Neutral lipids and dihydrotestosterone were removed with $100 \%$ iso-octane followed by $10 \%$ ethyl acetate: isooctane. We collected T with application of $20 \%$ ethyl acetate: isooctane. Fractions were dried at $35{ }^{\circ} \mathrm{C}$ under nitrogen, reconstituted in $500 \mu \mathrm{L}$ of phosphate-buffered saline, and stored at $4{ }^{\circ} \mathrm{C}$ overnight. Duplicate $\mathrm{T}$ fractions were incubated overnight with $3 \mathrm{H}-\mathrm{T}$ (5000 CPM) and antibody (1:500; \#T-3003; originally produced by Wien Laboratories, sold by Fitzgerald, Concord, MA; as in [33]). Unbound hormone was absorbed with dextran-coated charcoal, and the samples centrifuged at 3000 RPM for $25 \mathrm{~min}$. The supernatant was mixed with $3.5 \mathrm{~mL}$ of UltimaGold scintillation fluid (PerkinElmer, Shelton, CT) and counted on a Beckman LS 6500. We then adjusted values for volume and recovery efficiency and compared them to a standard curve run in triplicate ( 0.98 to $250 \mathrm{pg}$ T per tube).

\subsection{Statistical analyses}

We analyzed behavioral data using two different approaches. First, we considered the rates of display, locomotor, and foraging behavior across the entire observation period for each individual. Second, we considered the behaviors from each behavioral "bout" separately, with a bout defined as all behaviors that occurred between 2 min intervals of inactivity. In this approach, we averaged the number of display and locomotor behaviors across the bouts for each individual, (thus calculating a mean number of behaviors per bout rather than a rate per unit time for each individual), allowing us to weight each social interaction equally. On average, we observed 10.6 bouts per female, lasting $4.2 \mathrm{~min} /$ bout, and 12.6 bouts per male, lasting $7.6 \mathrm{~min} /$ bout.

\subsubsection{Analysis of total observations}

Because our reproductive behavior variables in males (proportion of time spent displaying, rate of dewlap extension, and rate of headbobs/push-ups) were strongly associated with one another, we used Principal Component Analysis (PCA) to reduce these three variables for use in subsequent analysis. The PCA extracted one principal component, hereafter called "display behavior" PC $(\lambda=2.74,91.2 \%$ variance explained) with high loadings for each of the three variables (display time $=0.92$, dewlap rate $=0.97$, head-bobs $/$ push- ups $=0.98$ ). We analyzed other behavioral rates (prey capture and total locomotion, measured as the sum of all runs, crawls, and jumps) individually. Pearson correlations were used to determine relationships among the testosterone, morphological, and behavioral variables.

As the structures are functionally linked, we also used PCA for the morphological traits associated with dewlap extension and for those regulating copulation. Using male dewlap-associated morphologies, two PCs were extracted (PC1 $\lambda=1.49,49.7 \%$ variance explained; PC2 $\lambda=0.98,32.6 \%$ variance explained). Structures that control dewlap extension loaded on PC1 (hereafter, "dewlap morphology" PC: cartilage area $=0.84, \mathrm{CH}$ muscle fiber size $=0.85$ ) and the measure related to overall dewlap size loaded highly on PC2 ("dewlap size" PC: cartilage length $=0.97)$. In a PCA with copulation-related morphologies, again two PCs were extracted (PC1 $\lambda=1.79,44.6 \%$ variance explained; PC2 $\lambda=1.21,30.2 \%$ variance explained). PC1 (hereafter, "hemipenis morphology" PC) had high loadings for hemipenis size (0.92) and RPM muscle fiber size (0.87), while PC2 ("ejaculate production" PC) had high loadings for seminiferous tubule size (0.75) and renal sex segment height $(-0.72)$. These PCs were used in multiple regression analyses to determine the relationships between morphology and behavior in males.

Parallel analyses to those described for males were performed with the data from females. A PCA on display behavioral traits extracted one PC ( $\lambda=1.97,65.7 \%$ variance explained) with high loadings for each of the three variables (display time $=0.92$, dewlap rate $=0.60$, head-bobs/push-ups $=0.80$ ). Pearson correlations among this "female display behavior" PC, locomotion rate, prey capture rate, and SVL were determined. We also performed a PCA on dewlap morphologies (cartilage length and area, $\mathrm{CH}$ fiber size) and extracted 2 PCs (PC1 $\lambda=1.57,52.4 \%$ variance explained; PC2 $\lambda=0.99,33.0 \%$ variance explained). PC1 ("female dewlap morphology PC") had high loadings for cartilage area (0.86) and $\mathrm{CH}$ fiber size (0.88), while PC2 ("female dewlap size PC") had a high loading for cartilage length (0.97). These two PCs and renal sex segment height were used in multiple regression analyses to determine the relationships between morphology and behavior in females.

Individual morphological traits were compared between males and females using ANOVA. To provide additional control for body size, these features were compared between the sexes using multivariate analyses of covariance (MANCOVA) with SVL as the covariate, as SVL was significantly correlated $(\mathrm{p}<0.05)$ with most of the traits measured. To compare behavioral traits between males and females, we used MANCOVA (again with SVL as the covariate) on display, locomotion, and prey capture rates.

\subsubsection{Analysis of behavioral bouts}

We analyzed behavioral bout data using the rates of display and locomotor behaviors per bout for each individual. We used the rate of all display behaviors (the sum of dewlap extensions and head-bobs/ push-ups) per bout as our measure of display. Total locomotion rate per bout was calculated as described above for total observational data. Prey capture data were not considered in the bout-based analysis, as prey capture occurred during relatively few bouts. We then used the morphological and testosterone measures described above, with parallel sets of analyses, to determine whether associations exist among these variables and the behavioral bout data.

\section{Results}

Behavioral bout data were strongly correlated with total observation data, as follows. For males, display behavior PC and displays per behavioral bout were positively correlated $(r=0.69, P=0.002)$, and movement rates in total observations and behavioral bouts were positively correlated $(r=0.67, p=0.002)$. Also for females, positive correlations also exist between display behavior PC and display rate 
per behavioral bout $(r=0.86, P<0.001)$, and movement rates in total observations and behavioral bouts $(r=0.84, p<0.001)$. Thus, we focus on reporting results from total observations, indicating where behavioral bout analyses differed.

\subsection{Behavior, morphology, and testosterone in males}

Males that performed more frequent displays had enhanced morphological traits associated with dewlap extension, but this relationship was not associated with body size (multiple regression of display behavior PC vs. dewlap morphology PC, dewlap size PC, and SVL: $\mathrm{R}^{2}=0.73, \mathrm{~F}_{3,14}=12.35, P<0.001$; Table 1 , top section). Analysis of individual dewlap morphology traits showed that cartilage length and area, but not $\mathrm{CH}$ fiber size, were associated with increased display behavior (Table 1, bottom section; Figs. 2 and 3a, b, e, and f). Analyses using total displays per behavioral bouts gave almost identical results, except that cartilage area was not associated with display behavior (results not shown).

Because of the strong associations between courtship and copulation in this species, we also examined relationships between courtship displays and copulatory morphology. Copulatory morphology was associated with display behavior, but not male body size (multiple regression of display behavior PC vs. hemipenis morphology PC, ejaculate production PC, and SVL: $R^{2}=0.51, F_{3,13}=4.42$, $P=0.024$, Table 2). Follow-up correlations between the variables with high loadings on ejaculate production PC and display behavior PC revealed that males that displayed more frequently had larger renal sex segments and smaller seminiferous tubules (display behavior PC and renal sex segment, $\mathrm{r}=0.48, P=0.044$, Fig. $3 c$ and $\mathrm{d}$; display behavior PC and seminiferous tubules, $\mathrm{r}=-0.58, P=0.015$, Fig. $3 \mathrm{~g}$ and $\mathrm{h}$ ). Analyses using total display rates during behavioral bouts revealed no relationships between copulation morphology and display behavior (results not shown).

Because we were concerned that plasma $T$ would be affected by the holding of animals prior to euthanasia, we examined whether $\mathrm{T}$ was correlated with restraint time in our dataset. No statistical relationship was detected $(r=-0.32, p=0.23)$. However, the two animals held for longer than $90 \mathrm{~min}$ had the lowest T levels, so these individuals were removed from subsequent analyses of $\mathrm{T}$. After this removal, there was still no relationship between $\mathrm{T}$ and restraint time $(r=0.03, p>0.9)$.

In the remaining dataset $(\mathrm{n}=14)$, plasma $T$ ( mean $=4.01 \mathrm{ng} / \mathrm{mL}$, $\mathrm{SE}=0.80$ ) was not significantly correlated with any of the morphological variables (SVL, mass, cartilage length or area, $\mathrm{CH}$ muscle fiber size, RPM muscle fiber size, hemipenis area, and renal sex segment height; all $P>0.1)$, except perhaps seminiferous tubule area $(r=0.54$, $P=0.060$ ). T was also not significantly correlated with locomotor or prey capture rates (both $P>0.5$ ), although increased T was associated with reduced display rates in total observation data ( $\mathrm{T}$ and display behavior PC: $r=-0.55, P=0.041)$, but this relationship was not significant in behavioral bout data $(r=-0.44, P=0.12)$.

\section{Table 1}

Relationships between behavior and dewlap morphologies. The top section indicates results from a multiple regression of display behavior PC vs. dewlap morphology PC, dewlap size PC, and body size (snout-vent length) in males. The bottom section lists results from individual regressions of display behavior PC vs. each morphological variable. Bold font indicates statistical significance.

\begin{tabular}{lrrr}
\hline & \multicolumn{1}{c}{ B } & \multicolumn{1}{l}{ T } \\
\hline Dewlap morph. PC & 0.610 & 3.93 & $\mathbf{0 . 0 0 2}$ \\
Dewlap size PC & 0.743 & 4.43 & $\mathbf{0 . 0 0 1}$ \\
SVL & -0.194 & -1.08 & 0.301 \\
& & & \\
Cartilage length & 0.787 & 4.88 & $<\mathbf{0 . 0 0 1}$ \\
Cartilage area & 0.337 & 2.30 & $\mathbf{0 . 0 4 0}$ \\
CH fiber size & 0.313 & 1.83 & 0.093 \\
\hline
\end{tabular}
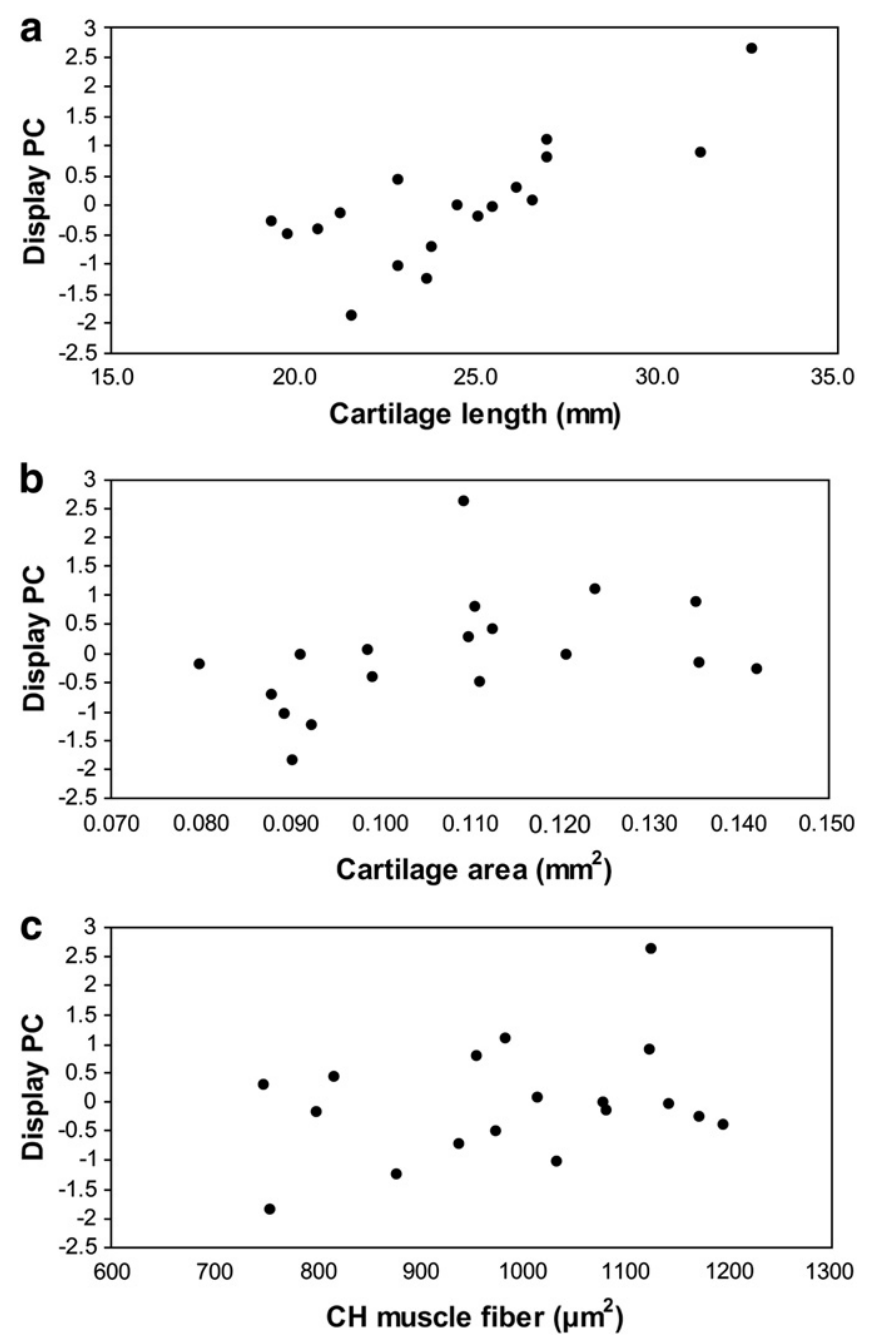

Fig. 2. Relationships between male display behavior PC (see text for details) and dewlap-associated morphological traits: a) second ceratobranchial cartilage length, b) cartilage area, c) ceratohyoid $(\mathrm{CH})$ muscle fiber size. Statistical results in Table 1, bottom section.

Correlations among behavioral traits showed that males performing more frequent displays also performed more locomotor behaviors (Table 3). The same relationship was also recovered in behavioral bout data $(r=0.73, P=0.001)$. However, SVL and prey capture rates were not correlated with locomotion, suggesting that increased locomotion was associated with social communication and territorial defense, and not foraging or body size in this group.

\subsection{Behavior and morphology in females}

Female display rate was not associated with the female dewlap morphology PCs or SVL (regression of female display behavior PC vs. dewlap morphology PC1 and PC2, and SVL: $\mathrm{R}^{2}=0.03, \mathrm{~F}_{3,16}=0.19$, $P=0.9)$. Renal sex segment height was also not associated with female display behavior PC $(\mathrm{r}=0.03, P=0.91)$. Results from analyses with behavioral bout data were identical (results not shown).

Correlations among behavioral traits showed that females that performed more frequent locomotor behaviors also exhibited higher rates of prey capture (Table 3). However, SVL or display rates were not correlated with locomotion, suggesting that increased locomotion was associated with foraging, and not territorial defense or body size. Again, results from analyses with behavioral bout data were identical (results not shown). 

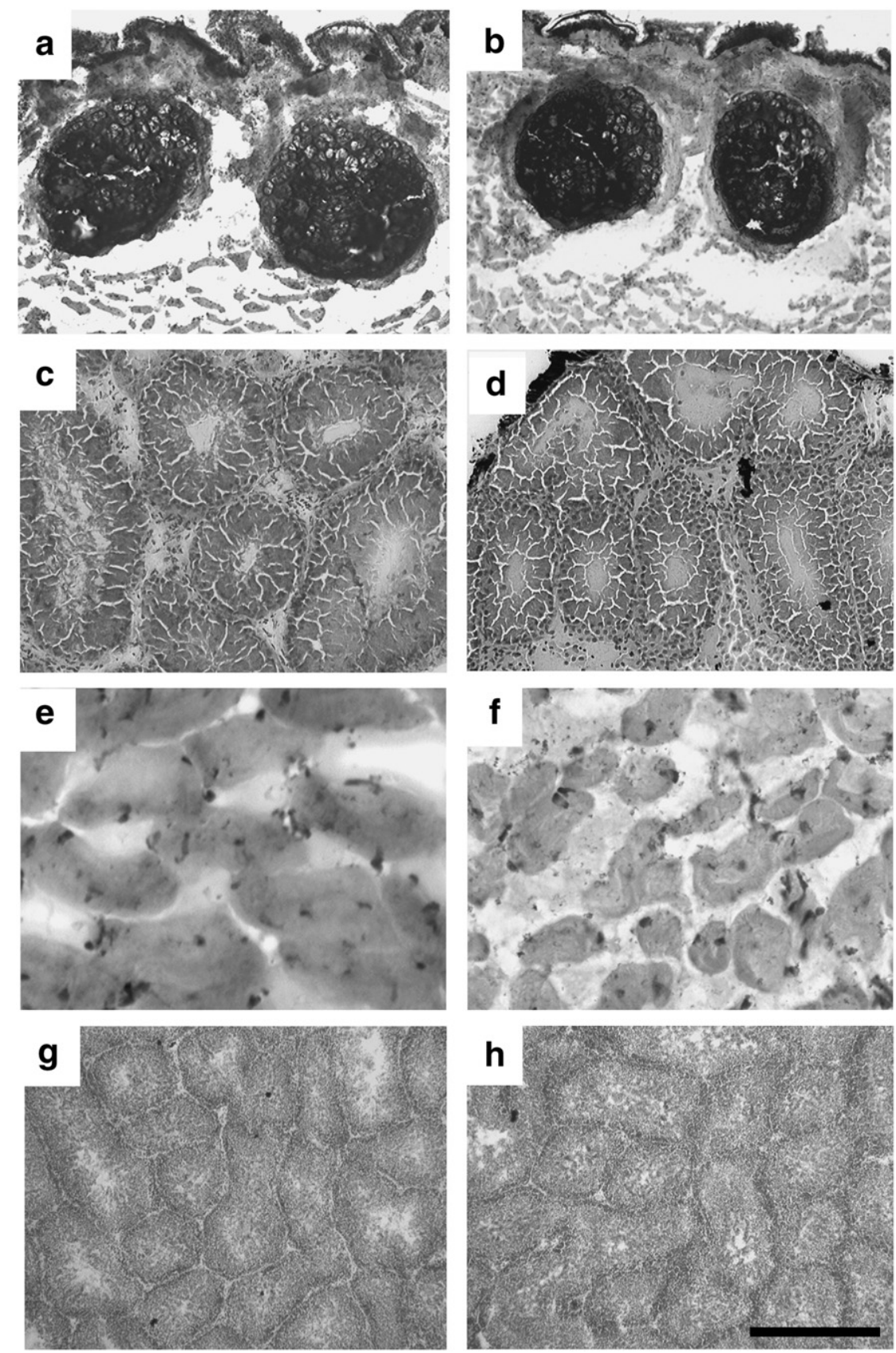

Fig. 3. Reproductive structures in high- (left column: a, c, e, g) and low-displaying (right column: b, d, f, h) males. Cross-sectional areas of cartilage (a, b) and renal sex segments (c, d) were positively correlated with display behavior. $\mathrm{CH}$ muscle fiber size (e, f) did not show a significant relationship, although a trend existed for males that displayed more to have larger fibers. Seminiferous tubules (g, h) were smaller in high displaying males. Scale bar $=330 \mu \mathrm{m}$ for (a) and (b); $180 \mu \mathrm{m}$ for (c) and (d); $60 \mu \mathrm{m}$ for (e) and (f); $500 \mu \mathrm{m}$ for (g) and (h).

\subsection{Morphology and behavior between males and females}

Males and females differed dramatically on all morphological traits associated with courtship and copulatory displays (Fig. 4). Cartilage

Table 2

Regression of display behavior PC vs. hemipenis morphology PC, ejaculate production PC, and body size (snout-vent length) in males. Bold font indicates statistical significance.

\begin{tabular}{lrrl}
\hline & \multicolumn{1}{c}{ B } & \multicolumn{1}{c}{ T } & $P$ \\
\hline Hemipenis morph. PC & 0.270 & 1.19 & 0.256 \\
Ejaculate production PC & -0.539 & -2.76 & $\mathbf{0 . 0 1 6}$ \\
SVL & 0.240 & 1.06 & 0.310 \\
\hline
\end{tabular}

length and cross-sectional area, as well as $\mathrm{CH}$ muscle fiber size were greater in males (all $\mathrm{F}_{1,34}>107, P<0.001$ ). The trachea was also larger in males than females $\left(F_{1,34}=26.4, P<0.001\right)$, although the magnitude of the sex difference was far smaller than for dewlap-associated

Table 3

Correlations among body size and behavioral traits. Male values are above the diagonal; female values below. Bold font indicates $P<0.01$; all other $P>0.09$.

\begin{tabular}{lrlll}
\hline & SVL & Display PC & Locomotion & Prey capture \\
\hline SVL & - & 0.41 & 0.22 & 0.02 \\
Display PC & 0.09 & - & $\mathbf{0 . 6 8}$ & -0.41 \\
Locomotion & 0.12 & 0.14 & - & -0.04 \\
Prey capture & -0.05 & 0.11 & $\mathbf{0 . 5 8}$ & - \\
\hline
\end{tabular}


a
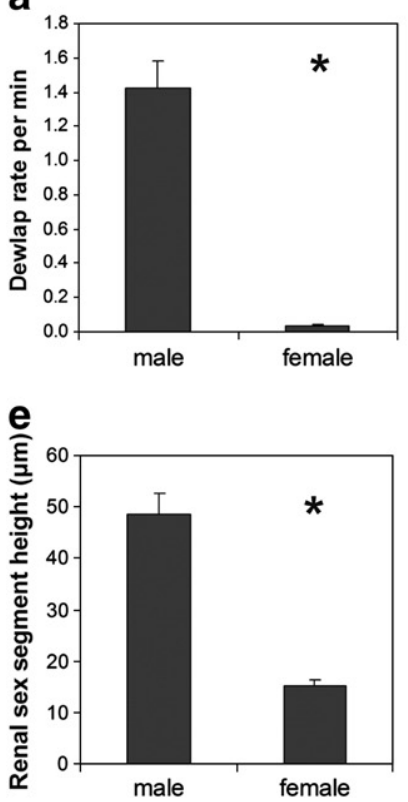

b

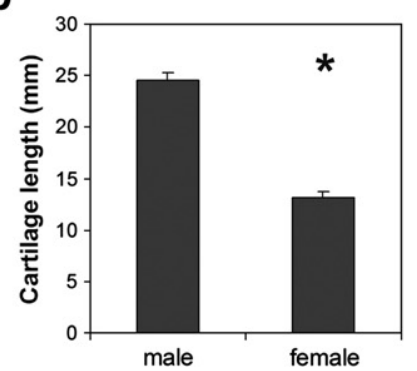

f

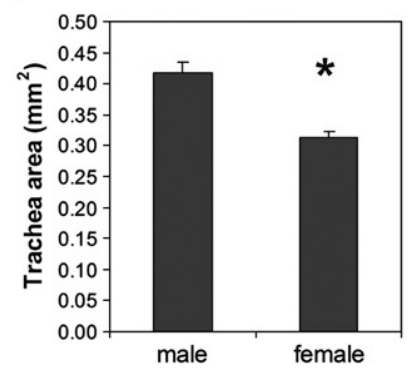

c

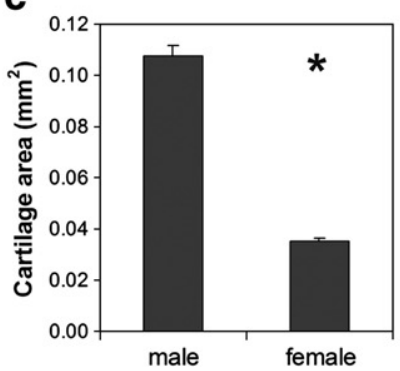

g

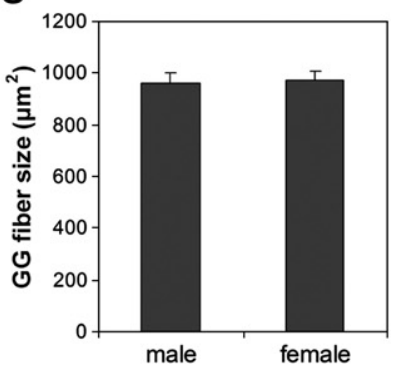

d

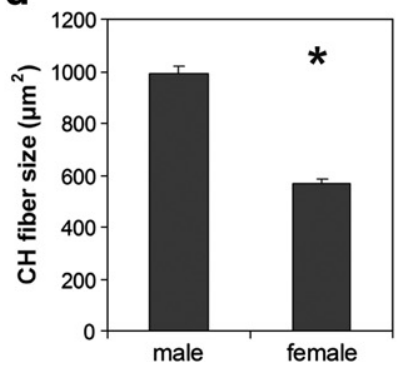

h

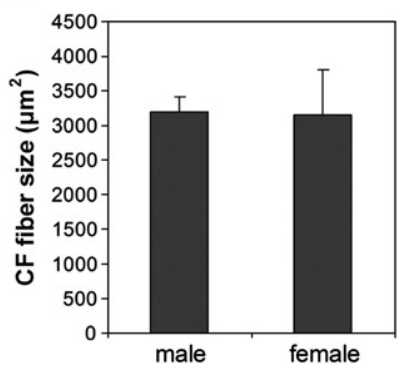

Fig. 4. Average values $(+\mathrm{SE})$ for male and female behavioral (a) and morphological (b-h) traits: a) dewlap display rate, b) second ceratobranchial cartilage length, c) second ceratobranchial cartilage area, d) ceratohyoid ( $\mathrm{CH}$ ) muscle fiber size, e) renal sex segment height, f) trachea area, g) genioglossus (GG) muscle fiber size, and h) caudiofemoralis ( $\mathrm{CF}$ ) muscle fiber size. Asterisks indicate $\mathrm{p}<0.001$

structures (Fig. 4; compare $\mathrm{f}$ to $\mathrm{b}-\mathrm{d}$ ). Fiber size in the control muscle, GG, did not differ between the sexes $\left(F_{1,34}=0.03, P=0.87\right)$ Comparisons of male and female copulatory morphology included only renal sex segments and CF fiber size, as females lack hemipenes and the muscles that control their movement [34]. The size of the renal sex segment, and androgen-stimulated organ, was greater in males than females $\left(\mathrm{F}_{1,34}=82.4, P<0.001\right)$, but fibers of the control $\mathrm{CF}$ muscle were equivalent in size in the two sexes $\left(F_{1,34}=0.00\right.$, $P=0.992$ ).

The data on control tissues suggest that the dimorphisms in reproductive structures are not simply due to differences in body size, but males are larger in both SVL and mass than females (ANOVA, both $\mathrm{F}>67, P<0.001$ ). To evaluate this issue more systematically, we conducted a series of comparisons for which SVL was controlled. MANCOVA comparing dewlap-associated morphologies (cartilage length and area, $\mathrm{CH}$ fiber size) between males and females showed that the sexes differed in these traits (sex: $\lambda_{3,34}=0.387, P<0.001$ ), but that SVL was not associated with sex differences (SVL: $\lambda_{3,34}=0.92$, $P=0.43$ ). MANCOVA comparing copulatory morphology (renal sex segments and $\mathrm{CF}$ fiber size) also revealed differences between the sexes (sex: $\lambda_{2,33}=0.79, P=0.022$ ), but no effect of body size (SVL: $\left.\lambda_{2,33}=0.95, P=0.46\right)$.

The sexes also differed in behavior. A MANCOVA comparing males and females revealed that when controlling for body size, males performed more dewlap extensions and marginally more head-bob displays (sex: $\lambda_{5,31}=0.62, P=0.009$; SVL: $\lambda_{5,31}=0.89, P=0.56$; ANCOVA results for each variable in Table 4). In analyses without SVL as a covariate, males still had higher dewlap extension and headbob rates, proportion of time displaying, and locomotion rates than females, but the sexes did not differ in prey capture rates (Table 4). Analyses using behavioral bout data revealed the same behavioral differences between the sexes (results not shown).

\section{Discussion}

The present study documents that a variety of structures associated with a reproductive display are larger in animals that perform this function more often, reinforcing common parallels between morphology and behavior. The results are not only largely consistent with previous laboratory studies of these lizards, but also illustrate that the neuromuscular and endocrine mechanisms underlying reproductive behavior in wild animals in their natural environments may interact in more complex ways than manipulations in the laboratory may reveal.

\subsection{Comparisons within the sexes}

Male green anoles that display more frequently have longer and thicker dewlap cartilage; males with larger dewlaps display those structures more often than other males. These relationships between behavior and morphology are not influenced by body size, as SVL was not associated with display behavior. Thus, the data demonstrate relatively specific relationships between structure and function.

In contrast, the size of $\mathrm{CH}$ muscle fibers was not associated with the rate of dewlap extension in the present study. This result differs from data using animals that were wild-caught, but behaviorally tested in the lab [33]. In that experiment, $\mathrm{CH}$ fiber size was greater in samples of male green anoles representing the top $18 \%$ compared to the bottom $18 \%$ of dewlap extension rates. In parallel, a significant positive correlation was detected between morphology and behavior in 29 individuals across the full range. Our results showed no correlation between $\mathrm{CH}$ and dewlap extension rate $(\mathrm{r}=0.33$, $P=0.19$ ), but analysis of our data using the methods of Neal and Wade [33] (comparing the top and bottom 18\% of males) produced

Table 4

Comparisons of male and female behavioral traits. ANCOVA (with SVL as the covariate) results comparing behaviors between the sexes. Values in parentheses show results from ANOVA without controlling for body size. Modified fonts indicate significant effects of sex.

\begin{tabular}{lrll}
\hline & \multicolumn{1}{l}{$\mathrm{F}$} & $\mathrm{df}$ & $P$ \\
\hline Proportion display time & $0.61(33.2)$ & 1,35 & $0.440(<\mathbf{0 . 0 0 1})$ \\
Dewlap extension rate & $9.33(91.4)$ & 1,35 & $\mathbf{0 . 0 0 4}(<\mathbf{0 . 0 0 1})$ \\
Head-bob rate & $3.96(54.6)$ & 1,35 & $0.054(<\mathbf{0 . 0 0 1})$ \\
Locomotion rate & $0.65(15.9)$ & 1,35 & $0.424(<\mathbf{0 . 0 0 1})$ \\
Prey capture rate & $0.002(0.30)$ & 1,35 & $0.961(0.590)$ \\
\hline
\end{tabular}


similar results $(\mathrm{t}=19.5, \mathrm{df}=7, P<0.001)$. Several explanations for the differences between the two types of analyses are possible. First, while males that display the most frequently may have larger $\mathrm{CH}$ fibers than males that display the least frequently, the relationship between dewlap rate and the size of muscle fibers producing this movement may be nonlinear. Differences between field and lab techniques could also account for the results. Neal and Wade [33] determined dewlap extension rate in the laboratory by introducing a novel female to a male in a small cage for $15 \mathrm{~min} /$ day over a period of 7 days. Here, we observed undisturbed behavior in the lizards' natural habitat for 64-178 min immediately before capture. Some lizards in this field study encountered females during the observation period, some encountered potential rival males, and for others the target of social interactions was not identified. Thus, the controlled setting of the laboratory environment may have allowed Neal and Wade [33] to detect more subtle relationships between morphology and behavior in a specific context than were possible in our field data.

Females exhibited no relationship between display behavior and dewlap morphology, indicating that in that sex, use of the dewlap is not associated with the size of the structures that support its movement. Because females perform these displays so rarely in comparison to conspecific males [20,21], it may be that these displays are not a sufficiently important component of female behavior to either cause or result from enhanced morphological structures. Females display the dewlap in their rare territorial encounters with other females, and occasionally to potential mates or predators, but at a rate that is generally at least one order of magnitude less than the display rates of males $[21,35,36]$. Also consistent with this idea, the variability among both dewlap-related structures and behaviors is lower in females than in males (see Fig. 4).

While copulatory structures are not directly used during courtship displays, reproduction comprises a full suite of behaviors such that a male often, and possibly necessarily, extends his dewlap prior to mounting and intromission (see Introduction). Therefore, one might expect some relationship between courtship behavior and morphology of copulatory structures. Here, we found that male display behavior is an indicator of some but not all aspects of copulation morphology. Display behavior is not related to size of the hemipenes or fibers of the muscles that control their movement, but associations exist between display rate and structures that produce the ejaculate. Specifically, males that display more have larger renal sex segments and smaller seminiferous tubules. Increased display might then relate to a male's increased sensitivity to androgens, even though these behaviors are negatively related to the level of circulating $\mathrm{T}$ itself. This idea is consistent with evidence from lab data documenting seasonal differences in the effectiveness of the same dose of $\mathrm{T}$ to both activate reproductive behaviors and increase renal sex segment size in male green anoles [37]. While the mechanism behind smaller seminiferous tubules in frequently-displaying lizards is unclear, examination of males in this study showed that all males were producing large numbers of mature sperm. One possibility is that males that spend more time courting females and/or defending their territories copulate less (although this seems unlikely [38,39]), and the decreased copulation relates to seminiferous tubule size. Further studies under conditions that allow detailed analysis of copulatory behavior are needed to address this issue.

Similarly, the apparent negative relationship between plasma $\mathrm{T}$ and display rate may result from males with higher baseline $\mathrm{T}$ having previously established dominance over those with lower $\mathrm{T}$ and thus no longer having the need to display frequently, a phenomenon that has been previously reported in this species [40]. Studies from multiple taxa, including some on red deer (Cervus elaphus), Harris sparrows (Zonotrichia querula), rats (Rattus norvegicus), and killifish (Kryptolebias marmoratus), have shown that animals with higher $\mathrm{T}$ are dominant to those with lower T [e.g., 41-44], and dyadic interactions in green anoles also show that dominant animals have higher $\mathrm{T}$ [45].
Another possibility is that animals with lower T may display more frequently to counterbalance performance deficiencies (lizards with decreased plasma T have lower bite-force capacity, but as a function of body size [12]) or to prime them for future takeovers of dominant male territories, such as the case in collared lizards [Crotophytus collaris; 46]. While these ideas warrant consideration, the results are consistent with Neal and Wade [33], who found no significant relationship between $\mathrm{T}$ and display rates, either across the full range of males or when comparing the top $18 \%$ to the bottom $18 \%$ of male dewlap rates. The results are collectively consistent with the idea that once the level is sufficient, having more $T$ does not enhance behavioral display rate. In addition, the lack of relationship between $\mathrm{T}$ and any measures of morphology in this study may reflect a threshold effect, such that a minimum level of circulating $T$ is necessary to maintain androgen-sensitive structures (including sizes of motoneuron somas, hemipenes, and RPM muscle fibers [32,47]) at a sufficient size to achieve reproduction, but more is not necessarily beneficial. This is true in other species, including hamsters (Mesocricetus auratus) [48] and rats[49], such that a threshold level of $\mathrm{T}$ is necessary for sexual behaviors, and any more $\mathrm{T}$ does not increase their display. These ideas should be further investigated in field studies of anoles designed specifically to address relationships of $\mathrm{T}$ with behavior and with morphology.

Plasma was not collected under ideal conditions in this study for those types of analyses, as T levels may change during restraint. Our primary goal was to maintain focused, extended behavioral observations so that these variables could be related to morphology. This approach resulted in the holding of some lizards prior to euthanasia while others were being observed. However, because we did not detect a correlation between holding time and T levels, it seems likely that any decrease in plasma $\mathrm{T}$ caused by stressful conditions occurred equivalently across individuals. The fact that there is a significant correlation between $\mathrm{T}$ levels and reduced behavioral displays supports the idea that meaningful individual variations in $\mathrm{T}$ existed.

\subsection{Behavior and morphology between males and females}

Males have larger morphologies than females for all dewlapassociated traits (cartilage length and area, $\mathrm{CH}$ muscle fiber size), and males perform display behaviors more frequently. Males also have larger renal sex segments than females, indicating increased sensitivity to $\mathrm{T}$ in breeding males compared to females [50]. Effects of adult $\mathrm{T}$ on cartilage morphology are not known, although $\mathrm{T}$ does increase cartilage length during juvenile development. Adult $\mathrm{T}$ does not modulate $\mathrm{CH}$ fiber size, but like the cartilage, juvenile $\mathrm{T}$ masculinizes morphology of this muscle [51]. Although there is evidence for yearly variation in dewlap area [52], it is not clear that this change is due to cartilage length, or influenced by T. Additionally, there are no effects of adult $\mathrm{T}$ treatment on muscle fiber size in the dewlap [32,37]. Collectively, the results suggest that, once fully developed, the morphology of structures regulating dewlap extension may not be affected by differing concentrations of circulating $T$. This idea suggests a dissociation between mechanisms directly regulating structure and function, as $\mathrm{T}$ is clearly required for display of the behavior (see Introduction).

The sexes do not differ in GG or CF fiber size, indicating some specificity in sexual dimorphism of reproductive structures. These findings are consistent with extensive previous work on green anoles (reviewed in [28]) and with results in species from diverse taxa, including zebra finches (Taeniopygia guttata), African clawed frogs (Xenopus laevis), and midshipmen fish (Poricthys notatus) showing that reproductive structures frequently used by males are enhanced in that sex [e.g., 3-7]. Importantly, the sex differences in the size of reproductive structures in this study cannot be explained by differences between the sexes in body size. In analyses that accounted for body size, male structures remained significantly larger than those 
in females, suggesting that the size of the structures are more likely associated with dramatic differences in their use. The present study and others [e.g., 20,36] have documented substantial sex differences in behavior. However, these results cannot indicate whether a causal relationship exists, or if it does, the direction of the relationship. It is possible that larger structures allow for more frequent use, or that frequent use of the structures enhances their size.

Interestingly, males performed displays and locomotor behavior more frequently than females in the present study, but the sexes did not differ in prey capture rates (Table 4). These behavioral differences may underlie different priorities between the sexes. Males that displayed more frequently than other males also performed more locomotor behaviors, but this was not related to prey capture or body size (Table 3). Thus, males were moving around the habitat more frequently to defend territories or court females, not to forage. Females, however, demonstrated a correlation between locomotion and prey capture (Table 3 ), so female movement was more likely related to foraging and not territory defense.

\subsection{Broader context and conclusions}

As both courtship and copulation are necessary components of reproduction for many animals, it is vital to study the behaviors and morphologies related to both of these functions in the same animals. These studies occur rarely in classic laboratory model systems, as most mammals exhibit stereotyped copulation but do not have easilystudied courtship displays, while many animals with elaborate courtship (birds, fish, and amphibians) lack copulatory organs and associated muscles. Lizards provide a particularly valuable opportunity to study both courtship and copulation behavior and morphology [28,53], and green anoles are a species readily studied in both the field and the lab.

Our observations of these animals in their natural habitats are largely consistent with findings from captive animals in laboratory conditions, but differences in the relationship between $\mathrm{CH}$ fiber size and behavior detected in the two settings highlight the need for both types of work. Field behavioral studies frequently provide ecological support for relationships identified in laboratory experiments, such as those conducted on dark-eyed juncos (Junco hyemalis) and Algerian mice (Mus spretus) [54,55], but laboratory environments are generally simplified versions of natural environments, providing, for example, less complex social interactions and more homogenous microhabitats [56]. On the other hand, laboratory settings allow for controlled experiments that are impossible to conduct in the field. Both types of studies are important for the complete understanding of behavioral traits. For example, the specific advantages offered by field and laboratory settings can be utilized to study behavioral, morphological, and genetic traits of two different populations, such as work done in prairie voles (Microtus orcheogaster) [57]. Additionally, work on song sparrows (Melospiza melodia) has demonstrated neurogenesis in wild birds, and used laboratory settings to examine more direct relationships between neurogenesis and behavior $[58,59]$. Thus, the controlled settings of laboratory studies and the natural settings offered by field studies both offer some clear advantages, but examinations of animal behavior and its underlying mechanisms in the laboratory and the field are necessary and complementary.

\section{Acknowledgments}

We are grateful for the logistical support of Jean Lafitte National Park, Barataria Preserve and for the advice of Manuel Leal, Danny Horton, Jerry Husak, Terry Christensen, and three reviewers. Tara Whittle assisted with data analysis. This work was supported by a Michigan State University Provost Office Postdoctoral Fellowship to MAJ, a Michigan State University McNair/Summer Research Oppor- tunities Program summer fellowship to JRV, and NSF IOS-0742833 to JW.

\section{Reference}

[1] Nelson RJ. An introduction to behavioral endocrinology. 3rd ed. Sunderland, Massachusetts: Sinauer; 2005.

[2] Cooke B, Hegstrom CD, Villeneuve LS, Breedlove MS. Sexual differentiation of the vertebrate brain: principles and mechanisms. Front Neuroendocrinol 1998;19: 323-62.

[3] Sassoon D, Kelley DB. The sexually dimorphic larynx of Xenopus laevis: development and androgen regulation. Am J Anat 1986;177:457-72.

[4] Kelley DB, Fenstemaker S, Hannigan P, Shih S. Sex differences in the motor nucleus of cranial nerve IX-X in Xenopus laevis: a quantitative Golgi study. J Neurobiol 1988; 19:413-29.

[5] Bass AH. Sounds from the intertidal zone-vocalizing fish. Bioscience 1990;40: 249-58.

[6] Arnold AP. Sexual differentiation of the zebra finch song system: positive evidence, negative evidence, null hypothesis, and a paradigm shift. J Neurobiol 1997;33:572-84.

[7] Wade J. Zebra finch sexual differentiation: the aromatization hypothesis revisited. Microsc Res Technol 2001;54:354-63.

[8] Adkins-Regan E. Hormones and animal social behavior. Princeton: Princeton University Press; 2005

[9] Pfaff DW, Arnold AP, Etgen AM, Farbach SF, Rubin RT. Hormones, brain and behavior. London: Academic Press; 2002.

[10] Tomaszycki ML, Banerjee SB, Adkins-Regan E. The role of sex steroids in courtship pairing, and pairing behaviors in the socially monogamous zebra finch. Horm Behav 2006;50:141-7.

[11] Wingfield JC, Moore MC. Hormonal, social, and environmental factors in the reproductive biology of free-living male birds. In: Crews D, editor. Psychobiology of Reproductive Behavior: an Evolutionary Perspective. Englewood Cliffs, N.J.: Prentice-Hall; 1987. p. 149-75.

[12] Husak JF, Irschick DJ, Meyers JJ, Lailvaux SP, Moore IT. Hormones, sexual signals, and performance of green anole lizards (Anolis carolinensis). Horm Behav 2007;52:360-7.

[13] McGlothlin JW, Whittaker DJ, Schrock SE, Gerlach NM, Jawor JM, Snajdr EA, et al. Natural selection on testosterone production in a wild songbird population. Am Nat 2010:175:687-701.

[14] Licht P. Environmental control of annual testicular cycles in lizard Anolis carolinensis. 1. Interactions of light and temperature. J Exp Zool 1967;165:505-16.

[15] Licht P. Environmental control of annual testicular cycles in lizard Anolis carolinensis. 2. Seasonal variations in effects of photoperiod and temperature on testicular recrudescence. J Exp Zool 1967;16:243-53.

[16] Crews D. Interrelationships among ecological, behavioral, and neuroendocrine processes in the reproductive cycle of Anolis carolinensis and other reptiles. In: Rosenblatt JS, Hinde RA, Beer CG, Busnel M-C, editors. Advances in the Study of Behavior. New York: Academic Press; 1980. p. 1-74.

[17] Ruby DE. Male breeding success and differential access to females in Anolis carolinensis. Herpetologica 1984;40:272-80.

[18] Jenssen TA, Lovern MB, Congdon JD. Field-testing the protandry-based mating system for the lizard, Anolis carolinensis: does the model organism have the right model? Behav Ecol Sociobiol 2001;50:162-72.

[19] Greenberg B, Noble GK. Social behavior of the American chameleon (Anolis carolinensis Voigt). Physiol Zool 1944;17:392-439.

[20] Nunez SC, Jenssen TA, Ersland K. Female activity profile of a polygynous lizard (Anolis carolinensis): evidence of intersexual asymmetry. Behavior 1997;134: 205-23.

[21] Johnson MA, Wade J. Courtship systems across nine Anolis lizard species: sexual dimorphisms in structure and function. Proc R Soc Lond B 2010;277:1711-9.

[22] Nicholson KE, Harmon LJ, Losos JB. Evolution of Anolis lizard dewlap diversity. PLoS One 2007;2:e274.

[23] Crews D. Effects of different components of male courtship behaviour on environmentally induced ovarian recrudescence and mating preferences in the lizard, Anolis carolinensis. Anim Behav 1975;23:349-56.

[24] Adkins E, Schlesinger L. Androgens and the social behavior of male and female lizard (Anolis carolinensis). Horm Behav 1979;13:139-52.

[25] Winkler SW, Wade J. Aromatase activity and regulation of sexual behaviors in the green anole lizard. Physiol Behav 1998;64:723-31.

[26] Rosen GJ, Wade J. The role of 5a-reductase in sexual behaviors of the green anole lizard. Physiol Behav 2000;69:487-98.

[27] Mason P, Adkins EK. Hormones and social behavior in the lizard, Anolis carolinensis. Horm Behav 1976;7:75-86.

[28] Wade J. Current research on the behavioral neuroendocrinology of reptiles. Horm Behav 2005;48:451-60.

[29] Bels VL. The mechanism of dewlap extension in Anolis carolinensis (Reptilia: Iguanidae) with histological analysis of the hyoid apparatus. J Morphol 1990;206: 225-44.

[30] Font E, Rome LC. Functional morphology of dewlap extension in the lizard Anolis equestris (Iguanidae). J Morphol 1990;206:245-58.

[31] Arnold EN. Variation in the cloacal and hemipenial muscles of lizards and its bearing on their relationships. Symp Zool Soc Lond 1984;52:47-85.

[32] O'Bryant EL, Wade J. Sexual dimorphisms in a neuromuscular system regulating courtship in the green anole lizard: effects of season and androgen treatment. J Neurobiol 1999;40:202-13. 
[33] Neal JK, Wade J. Androgen receptor expression and morphology of forebrain and neuromuscular systems in male green anoles displaying individual differences in sexual behavior. Horm Behav 2007;52:228-36.

[34] Ruiz CC, Wade J. Sexual dimorphisms in a copulatory neuromuscular system in the green anole lizard. J Comp Neurol 2002;443:289-97.

[35] Andrews TJ, Summers CH. Aggression, and the acquisition and function of social dominance in female Anolis carolinensis. Behaviour 1996;133:1265-79.

[36] Jenssen TA, Orrell KS, Lovern MB. Sexual dimorphisms in aggressive signal structure and use by a polygynous lizard, Anolis carolinensis. Copeia 2000;1:140-9.

[37] Neal JK, Wade J. Courtship and copulation in the adult male green anole: effects of season, hormone and female contact on reproductive behavior and morphology. Behav Brain Res 2007;177:177-85.

[38] Husak JF, Fox SF, Lovern MB, Van Den Bussche RA. Faster lizards sire more offspring: sexual selection on whole-animal performance. Evolution 2006;60: 2122-30.

[39] Husak JF, Fox SF, Van Den Bussche RA. Faster male lizards are better defenders not sneakers. Anim Behav 2008;75:1725-30.

[40] Qualls CP, Jaeger RG. Dear enemy recognition in Anolis carolinensis. J Herpetol 1991;25:361-3.

[41] Lincoln GA, Guinness F, Short RV. The way in which testosterone controls the social and sexual behavior of the red deer stag (Cervus elaphus). Horm Behav 1972;3:375-96.

[42] Rohwer S, Rohwer FC. Status signaling in Harris sparrows: experimental deceptions achieved. Anim Behav 1978;26:1012-22.

[43] Albert DJ, Walsh ML, Gorzalka BB. Testosterone removal in rats results in a decrease in social aggression and a loss of social dominance. Physiol Behav 1986;36:401-7.

[44] Early RL, Hsu Y. Reciprocity between endocrine state and contest behavior in the killifish, Kryptolebias marmoratus. Horm Behav 2008;53:442-51.

[45] Greenberg N, Crews D. Endocrine and behavioral responses to aggression and social dominance in the green anole lizard, Anolis carolinensis. Gen Comp Endocrinol 1990;77:246-55.

[46] Baird TA, Hews DK. Hormone levels in territorial and non-territorial male collared lizards. Physiol Behav 2007;92:755-63.

[47] Holmes MM, Wade J. Seasonal plasticity in the copulatory neuromuscular system of green anole lizards: a role for testosterone in muscle but not motoneuron morphology. J Neurobiol 2004;60:1-11.
[48] Meek LR, Romeo RD, Novak CM, Sisk CL. Actions of testosterone in prepubertal and postpubertal male hamsters: dissociation of effects on reproductive behavior and brain androgen receptor immunoreactivity. Horm Behav 1997;31:75-88.

[49] Damassa DA, Smith ER, Tennent B, Davidson JM. The relationship between circulating testosterone levels and male sexual behavior in rats. Horm Behav 1977;8:275-86.

[50] Lovern MB, McNabb FMA, Jenssen TA. Developmental effects of testosterone on behavior in male and female green anoles (Anolis carolinensis). Horm Behav 2001;39:131-43.

[51] Lovern MB, Holmes MM, Fuller CO, Wade J. Effects of testosterone on the development of neuromuscular systems and their target tissues involved in courtship and copulation in green anoles (Anolis carolinensis). Horm Behav 2004;45:295-305.

[52] Irschick DJ, Ramos M, Buckley C, Elstrott J, Carlisle E, Lailvaux SP, et al. Are morphology-performance relationships invariant across different seasons? A test with the green anole lizard (Anolis carolinensis). Oikos 2006;114:49-59.

[53] Greenberg N. A neuroethological study of display behavior in the lizard Anolis carolinensis (Reptilia, Lacertilia, Iguanidae). Am Zool 1977;17:191-201.

[54] Castro JM, Nolan Jr V, Ketterson ED. Steroid hormones and immune function: experimental studies in wild and captive dark-eyed juncos (Junco hyemalis). Am Nat 2001;157:408-20.

[55] Cassaing J, Cervera S, Isaac F. Laboratory and field evidence of paternal care in the Algerian mouse (Mus spretus). J Ethol 2009;28:7-13.

[56] Fusani L, Canoine V, Goymann W, Wikelski M, Hau M. Difficulties and special issues associated with field research in behavioral neuroendocrinology. Horm Behav 2005;48:484-91.

[57] Ophir AG, Phelps SM, Sorin AB, Wolff JO. Morphological, genetic, and behavioral comparisons of two prairie vole populations in the field and laboratory. J Mammal 2007;88:989-99.

[58] Tramontin AD, Brenowitz EA. A field study of seasonal neuronal incorporation into the song control system of a songbird that lacks adult song learning. J Neurobiol 1999;40:316-26.

[59] Reeves BJ, Beecher MD, Brenowitz EA. Seasonal changes in avian song control circuits do not cause seasonal changes in song discrimination in song sparrows. J Neurobiol 2003;57:119-29. 